\title{
Gate-assisted phase fluctuations in all-metallic Josephson junctions
}

\author{
J. Basset $\odot,{ }^{*}$ O. Stanisavljević, M. Kuzmanović $\odot,{ }^{\dagger}$ J. Gabelli $\odot$, C. H. L. Quay, J. Estève $\odot$, and M. Aprili \\ Université Paris-Saclay, Centre National de la Recherche Scientifique, Laboratoire de Physique des Solides, 91405 Orsay, France
}

(Received 12 July 2021; accepted 27 October 2021; published 10 December 2021)

\begin{abstract}
We study the reduction of the supercurrent by a gate electrode in a purely metallic superconductor-normal metal-superconductor Josephson junction by performing, on the same device, a detailed investigation of the gate-dependent switching probability together with the local tunneling spectroscopy of the normal metal. We demonstrate that high energy electrons leaking from the gate trigger the reduction of the critical current which is accompanied by an important broadening of the switching histograms. The switching rates are well described by an activation formula including an additional term accounting for the injection of rare high energy electrons from the gate. The rate of electrons obtained from the fit remarkably coincides with the independently measured leakage current. Concomitantly, a negligible elevation of the local temperature in the junction is found by tunneling spectroscopy which excludes stationary heating induced by the leakage current as a possible explanation of the reduction of the critical current. This incompatibility is resolved by the fact that phase dynamics and thermalization effects occur at different timescales.
\end{abstract}

DOI: 10.1103/PhysRevResearch.3.043169

\section{INTRODUCTION}

Understanding the dynamics of quasiparticle excitations in a superconductor and how they interact with the superconducting condensate is important for understanding dissipation mechanisms responsible for decoherence in superconducting devices [1,2]. For example, thermally induced phase slips of the superconducting order parameter or absorption of electromagnetic radiation locally suppress superconductivity, which produces quasiparticles and hence decoherence [3-6]. Whereas the injection of low energy quasiparticles (meV) in mesoscopic superconductors has been extensively studied [7-10], little is known about the injection of high energy $(\mathrm{eV})$ quasiparticles [11], especially in the limit of few quanta.

Recently, it has been observed that the critical current of a superconducting weak link formed by a mesoscopic nanowire or constriction [12-19] can be suppressed by applying a voltage bias to a side gate in the vicinity of the weak link. This all-metal-based superconducting device, which at first sight seems to operate like a semiconducting field effect transistor, could be used in quantum technologies as a tunable inductance or switch provided dissipation is demonstrated to be low [20-22]. Beyond such practical implications, the understanding of this unexpected effect is attracting a lot of attention. In

\footnotetext{
*julien.basset@universite-paris-saclay.fr

${ }^{\dagger}$ Present address: QTF Centre of Excellence, Department of Applied Physics, Aalto University School of Science, P.O. Box 15100, 00076 Aalto, Finland.

${ }^{\ddagger}$ marco.aprili@universite-paris-saclay.fr

Published by the American Physical Society under the terms of the Creative Commons Attribution 4.0 International license. Further distribution of this work must maintain attribution to the author(s) and the published article's title, journal citation, and DOI.
}

particular, even though it is still disputed, there is indirect evidence that the leakage current of high energy electrons from the gate plays a major role in reducing the critical currents of mesoscopic constrictions [11,23-25].

The goal of this paper is to investigate the transition regime in which a supercurrent reduction is induced by a small $(\approx 10 \mathrm{fA})$, high energy, quasiparticle leakage rate which already demonstrates a sizable and puzzling reduction of the supercurrent. In particular, we make use of complementary tools (tunnelling spectroscopy [26-28] and switching probability histograms) to study the gate-induced reduction of the supercurrent in a superconductor-normal metal-superconductor (SNS) Josephson junction. A side gate allows us to attenuate the critical current of the junction in a way similar to previous studies [11-19,23-25]. The SNS junction contains a tunnel electrode to perform tunnel spectroscopy of the proximitized region. The current leakage from the gate to the normal metal is composed of rare $\left(\Gamma_{L}=I_{L} / e \approx\right.$ $\left.2 \times 10^{4} \mathrm{~s}^{-1}\right)$ and high energy $(\approx 5 \mathrm{eV})$ electrons. It is accompanied by a large broadening of the switching histograms [17] while, at the same time, a small elevation $(\approx 48 \mathrm{mK}$ for $6.8 \mathrm{eV})$ of the local time-averaged electron temperature is found by tunneling spectroscopy, excluding a simple overheating scenario [11]. Instead, each high energy (hot) electron reaching the junction undergoes a series of energy relaxation events including the emission of optical and acoustic phonons [25] that can effectively be described as a large temperature spike $T_{0} \gg\left\{T_{e}, T_{\varphi}, T\right\}$ (electron, phase, and bath temperatures, respectively) at the junction stage. Those spikes lead to an enhancement of the switching rate for small bias currents. In the limit of low leakage currents, they are independent events and too rare to significantly elevate the time-averaged electron temperature in the junction. The values of the leakage current obtained from this model match the leakage current measured independently with high resolution, confirming the soundness 

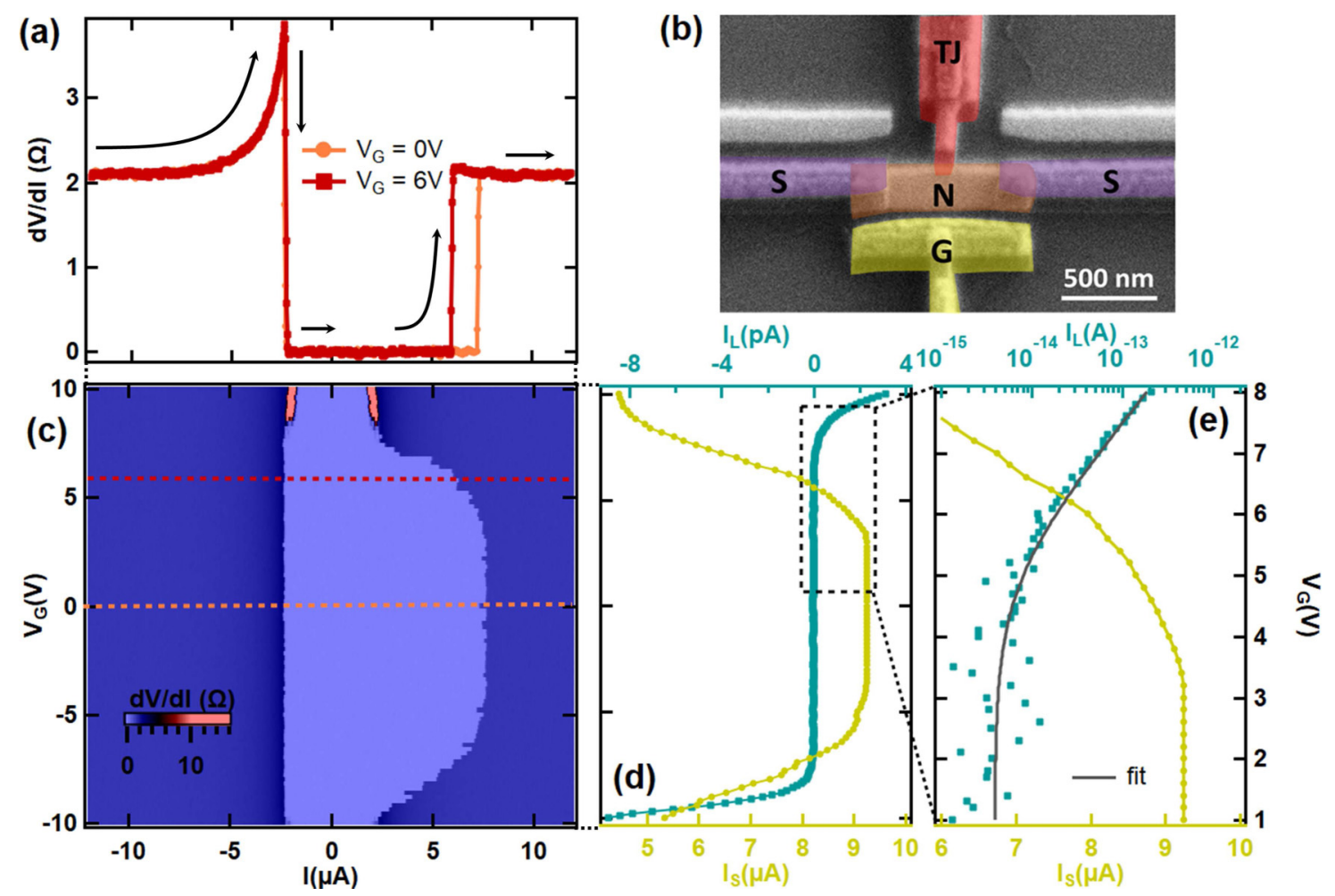

FIG. 1. Switching and leakage currents in a gated Josephson junction. (a) Differential resistance vs current bias $I$ for the two gate voltages $V_{G}$ indicated by dashed lines in (c). (b) Colorized scanning electron microscope picture of the sample showing the superconducting contacts $\mathrm{S}$, the normal metal $\mathrm{N}$, the tunnel junction electrode TJ, and the gate $\mathrm{G}$. (c) Colormap of the differential resistance as a function of $I$ and $V_{G}$. (d) Leakage current $I_{L}$ and mean switching current $I_{S}$ as a function of $V_{G}$. (e) Zoom of (d) in the region of low leakage currents. See Supplemental Material [35] for the fit. (a) and (c) share the same $x$ axis. (c) and (d) share the same $y$ axis.

of our model. The proposed scenario is similar to the hotspots created by photons acting on superconducting nanowires or Josephson junction based single photon detectors [29-34].

The paper is organized as follows. After presenting the sample fabrication and the measurement setup in Sec. II, we investigate the static properties of the gating effect in Sec. III where we study critical current reduction and leakage current and perform tunnel spectroscopy. We then analyze the phase dynamics in Sec. IV that we compare to a model of independent high energy quasiparticles reaching the weak link. Discussion of the results, including comparison to other experiments, is given in Sec. V.

\section{SAMPLE FABRICATION AND EXPERIMENTAL SETUP}

The sample consists of a SNS Josephson junction tunnel coupled to a superconducting electrode and a side gate $(\mathrm{G})$. All superconducting electrodes are made of $\mathrm{Al}$ while the normal metal is $\mathrm{Cu}$. The tunnel junction (TJ) is used to probe the density of states and the local temperature in $N$ [27]. A typical sample is shown in Fig. 1(b). The tunnel junction surface is roughly $110 \times 80 \mathrm{~nm}$ and the gate is located $50 \mathrm{~nm}$ away from the SNS junction. The device is fabricated in a single step $e$-beam lithography followed by a three angle $e$-gun evaporation technique. We first deposit a thin 10-nm layer of Al with a positive angle [red in Fig. 1(b)]. This layer is then oxidized in pure oxygen to form a good tunnel barrier with $45 \mathrm{~nm}$ of copper evaporated at zero angle (orange) just after oxidation. A third evaporation, at a negative angle, of $100 \mathrm{~nm}$ of $\mathrm{Al}$ defines the superconducting contacts (violet) as well as the gate electrode (yellow) necessary to tune the supercurrent across the SNS junction. The samples are cooled down in a dilution refrigerator at $110-\mathrm{mK}$ base temperature. Each contact of the SNS and tunnel junctions is connected through dissipative, highly filtered, and closely packed twisted pairs. Of note, the gate electrode is independently connected to a semirigid microwave cable to avoid stray leakage currents with the measurement lines (see Supplemental Material for details [35]). The SNS junction normal state resistance is $R_{n} \approx$ $2 \Omega$ whereas the tunnel junction resistance is $R_{t} \approx 4 \mathrm{M} \Omega$. Therefore the tunnel junction is noninvasive for voltages lower than $0.5 \mathrm{mV}$ where the current reaches roughly $100 \mathrm{pA}$. From the temperature dependence of the tunneling spectroscopy and the geometry, we estimate the Thouless energy $E_{\mathrm{Th}}=$ $\hbar D / L^{2}=\hbar / \tau_{D} \approx 14.5 \mu \mathrm{eV}$ and a diffusion time $\tau_{D} \approx 46 \mathrm{ps}$.

\section{STATIONARY PROPERTIES}

\section{A. Gate-dependent supercurrent and leakage current}

We first address the gate-induced change of the mean switching current, $I_{s}$, by measuring the differential resistance $d V / d I(I)$ as a function of the current $I$ and gate voltage $V_{G}$. The results are shown as a colormap in Fig. 1(c) with line cuts in Fig. 1(a). As in previous reports [12-19], no gate dependence of $I_{S}$ is observed below a threshold value which in our case is about $\approx \pm 4 \mathrm{~V}$. For $\left|V_{G}\right|>4 \mathrm{~V}$ and up 

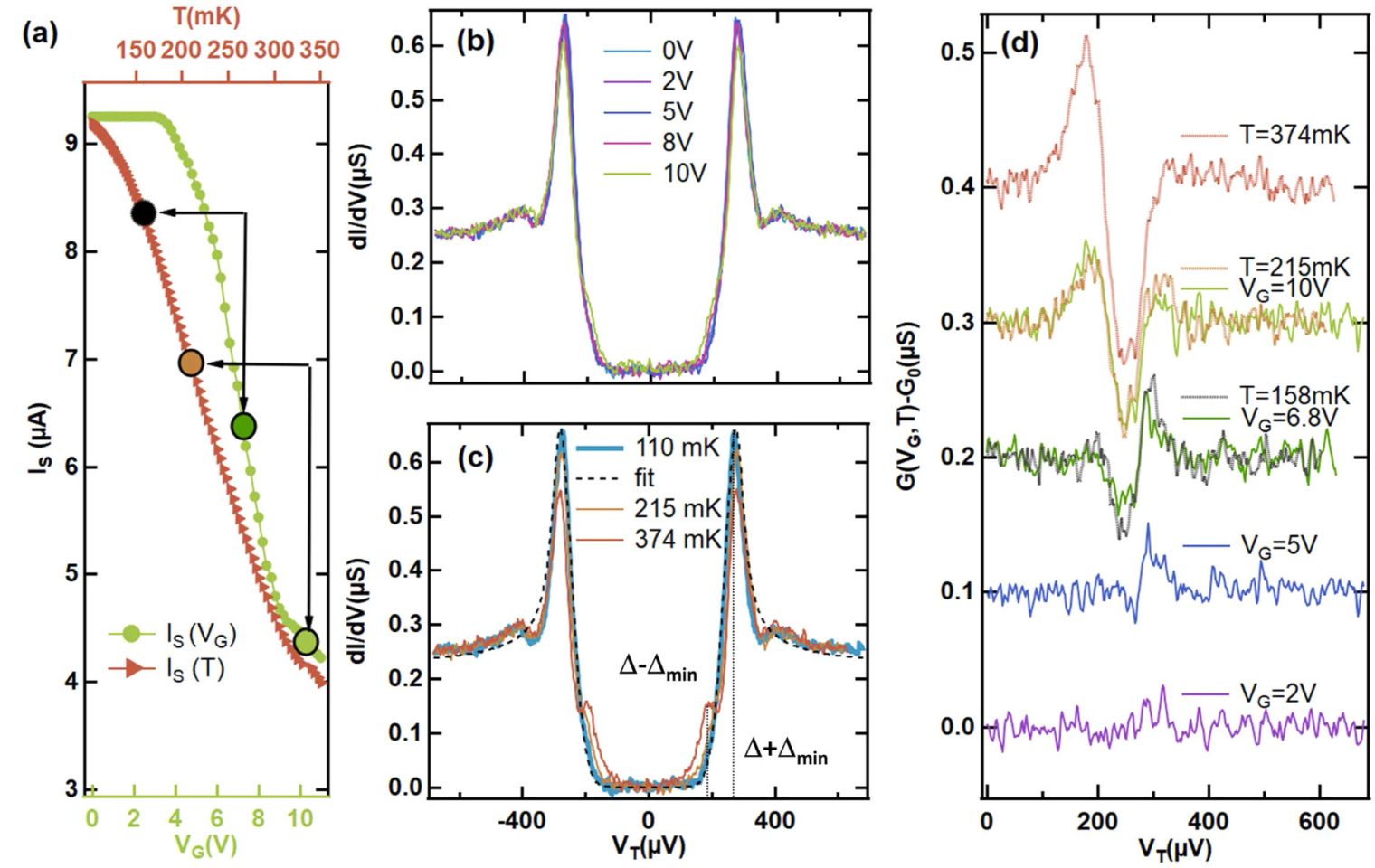

FIG. 2. Local thermometry using tunneling spectroscopy. (a) Mean switching current $I_{S}$ as a function of the bath temperature $T$ (as measured by a calibrated $\mathrm{RuO}_{2}$ resistor anchored to the mixing chamber next to the sample) and gate voltage $V_{G}$. (b), (c) Differential conductance of the tunnel junction at different (b) gate voltages and (c) temperatures. The in-gap feature observed at $T=374 \mathrm{mK}$ is due to thermally excited quasiparticles through the minigap in N. (d) Numerical difference $G\left(V_{G}, T\right)-G_{0}$ (see text) for different temperatures and gate voltages. The comparison between thermal and gate voltage effects indicates that the decrease in mean switching current cannot be accounted for quantitatively by overheating.

to $\left|V_{G}\right| \approx 9 \mathrm{~V}$ the switching current is reduced by about $50 \%$ while the retrapping current, the current at which the voltage drop snaps back to zero, $I_{r}$, is unaffected. Above $\left|V_{G}\right| \approx 9 \mathrm{~V}$ the hysteresis in the I-V characteristics disappears and $I_{s}=I_{r}$. The switching current is suppressed further by increasing $V_{G}$. We separately measure with high accuracy the leakage current from the gate, $I_{L}$, as a function of the gate voltage [Figs. 1(d) and $1(\mathrm{e})$ ]. Such precision was achieved using an automated differential measurement technique between gate on and off as explained in the Supplemental Material [35]. These data demonstrate a clear correlation between the critical current reduction and the leakage current.

\section{B. Tunneling spectroscopy}

In order to show that the reduction of the switching current is not due to the heating of the junction ( $\mathrm{N}$ part), i.e., an elevation of the local stationary (time-averaged) electronic temperature $T_{e}$, we first measure its temperature and gate dependence $I_{S}(T)$ and $I_{S}\left(V_{G}\right)$ shown in Fig. 2(a). We then probe, for a second time, the increase of $T_{e}$ due to the gate voltage by tunneling spectroscopy. The comparison of the temperature and gate datasets allows us to conclude that overheating is, in the limit of small leakage current, a negligible source of reduction of the critical current.

To access experimentally the electronic temperature of the normal metal region, we perform tunnel spectroscopy by measuring the nonlinear conductance of the tunnel contact coupled to the $\mathrm{N}$ part of the SNS junction [see Fig. 1(b)] as a function of gate voltage [Fig. 2(b)] and temperature [Fig. 2(c)]. In this geometry, the quasiparticle current that flows through the junction is given by

$I_{T}(V)=\frac{1}{e R_{t}} \int d E N_{S}(E) N_{N}(E-e V)\left[f_{N}(E-e V)-f_{S}(E)\right]$

where $N_{S}$ is the Al BCS density of states and $N_{N}$ is the density of states in $\mathrm{N}$ as modified by the proximity effect. $f_{S}$ and $f_{N}$ are the distribution functions. Assuming that $f_{S}$ and $f_{N}$ are the Fermi-Dirac distribution functions at the bath temperature and at $T_{e}$, respectively, Eq. (1) predicts that a rounding of the distribution function, due to an increase of $T_{e}$, shall be visible for a voltage bias of the tunnel junction $e V_{T} \approx \Delta+$ $\Delta_{\text {min }}$ where $\Delta=195 \mu \mathrm{eV}$ is the Al superconducting gap and $\Delta_{\text {min }} \approx 3.1 E_{\mathrm{Th}} \approx 45 \mu \mathrm{eV}$ is the minigap induced in $\mathrm{N}$ [26].

We show in Fig. 2(b) the differential conductance of the tunnel probe as a function of bias voltage $V_{T}$ for different gate voltages. At the lowest temperature, and only in the extreme case of high gate voltage $V_{G}=10 \mathrm{~V}$, a small modification of the differential conductance is visible corresponding to a slight overheating of $\mathrm{N}$.

To estimate $\Delta T$, we compare these gate-dependent datasets to the ones obtained as a function of the bath temperature reported in Fig. 2(c). The temperature dependence is well captured by Eq. (1). In particular, the high temperature curve 

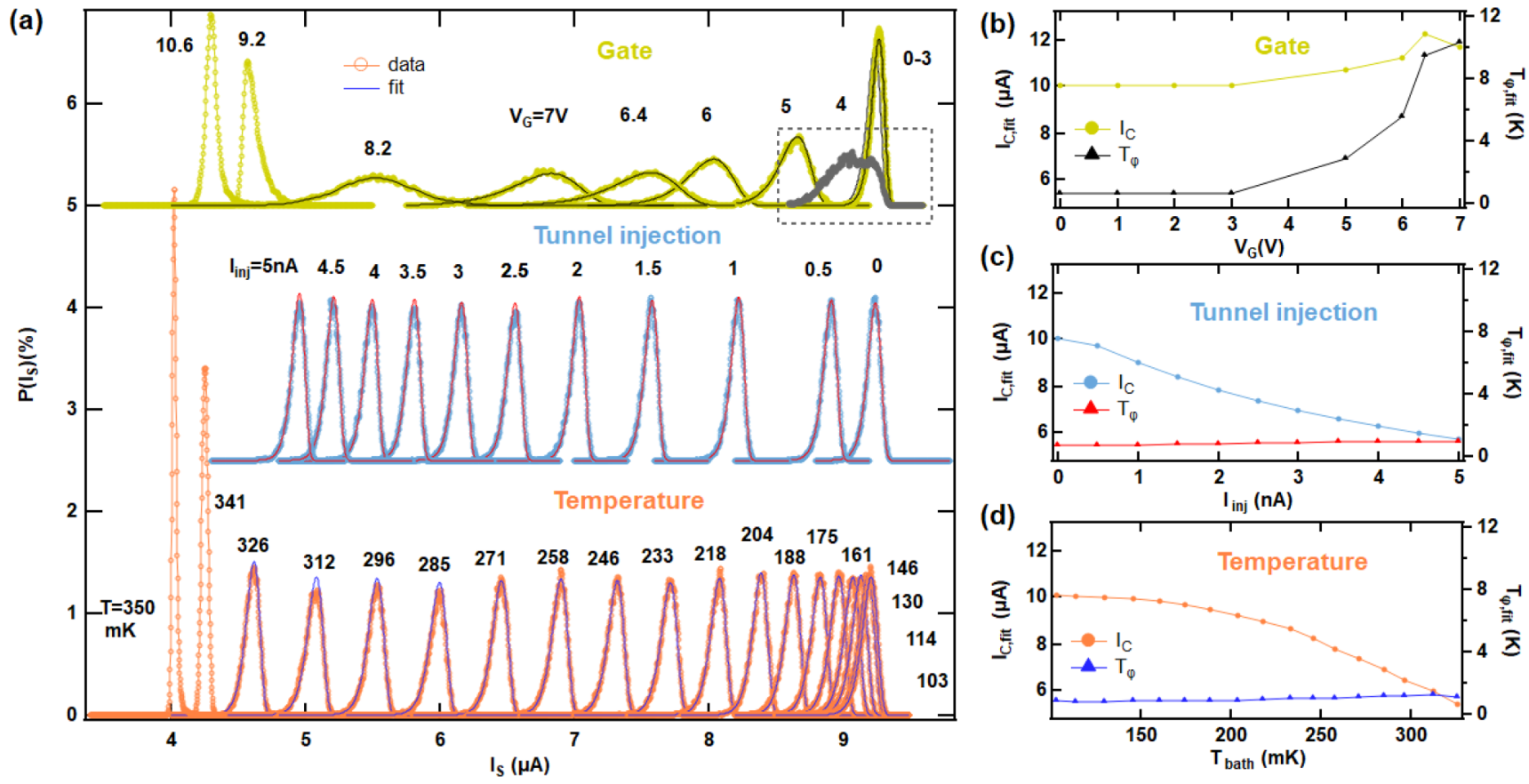

FIG. 3. Phase dynamics. (a) Evolution of the switching probability histograms ( $v=37 \mathrm{~Hz}, M=12000$ ) and their fits using Eq. (3) with respect to temperature, tunnel current injection, and gate voltage. While temperature and tunnel injection show similar behavior, the gate dependence exhibits a larger broadening of the histograms for the equivalent mean switching current values. At high temperature, the narrowing of the histogram is due to the enhancement of the dissipation. The dashed box highlights the transition regime where Eq. (3) cannot explain the data. Evolution of the fitting parameters $I_{C, f i t}$ and $T_{\varphi}$ is also shown with respect to (b) gate voltage, (c) tunnel current injection, and (d) temperature with the exact same scales.

exhibits a peak in the differential conductance at $e V_{T}=\Delta-$ $\Delta_{\min }$ when the bath temperature becomes comparable with the minigap $\Delta_{\min }$. This peak comes from thermal quasiparticles in N. Because the deviations from equilibrium are small we show in Fig. 2(d) the numerical difference $G\left(V_{G}, T\right)-G_{0}$ where $G_{0}=G\left(V_{G}=0 \mathrm{~V}, \mathrm{~T}=110 \mathrm{mK}\right)$. This difference shows that gating starts to raise the temperature $T_{e}$ from $V_{G} \approx 5 \mathrm{~V}$. We see experimentally that for $V_{G}=6.8 \mathrm{~V}$ the increase in $T_{e}$ is about 50 and $100 \mathrm{mK}$ for $V_{G}=10 \mathrm{~V}$ as can be seen through the perfect overlaps shown in Fig. 2(d). At the same time, even though the tunneling data coincide, strong differences are observed in the mean switching current [see circles and arrows in Fig. 2(a) that show the positions where the electronic temperatures are equivalent, either due to gate or temperature, but not the switching currents], which is a first hint that the large gate-induced supercurrent reduction observed experimentally cannot be explained by a simple overheating $[11,36]$.

We now relate the small raise of temperature to the leakage current. For that we estimate the increase $\Delta T$ in the average electron temperature $T_{e}$ in the junction due to a flow of high energy electrons. To do so we assume that each temperature spike has an amplitude $T_{0}$ with an exponential decay rate of $1 / \tau_{R}$ (with $\tau_{R}$ the energy relaxation time) and a repetition rate $\Gamma_{L}$. Taking the time-averaged value of the spikes gives $\Delta T \approx$ $T_{0} \tau_{R} \Gamma_{L}$. At $V_{G}=10 \mathrm{~V}$ where the leakage current reaches $I_{L}=e \Gamma_{L}=3 p A$, we get $\Delta T \approx 100 \mathrm{mK}$ and $T_{0} \approx 2 \mathrm{~K}$ is the temperature spike generated by a single high energy quasiparticle according to $T_{0}=(2 E / \gamma \Omega)^{0.5}$ where $E \approx e V_{G}, \gamma$ is the Sommerfeld constant of $\mathrm{N}$, and $\Omega$ is the diffusion volume $\left(\approx 8 \times 10^{-15} \mathrm{~cm}^{3}\right)$ [37]. The relaxation time in our case is $\tau_{R} \approx 2.5 \mathrm{~ns}$, the time for the high energy quasiparticles to reach the reservoirs. The volume $\Omega$ includes both the copper junction and also the small $\mathrm{Al}$ contacts until the larger contacts sink the electrons. The small Al leads need to be taken into account because of the high energy of the electrons which are not bound to copper [38,39]. This crude estimation of $T_{e}$ for $V_{G}=10 \mathrm{~V}$ is comparable to the value obtained by tunneling spectroscopy.

\section{SWITCHING DYNAMICS}

We have seen in the previous section that the effect of the gate on the time-averaged electronic temperature is negligible while the switching current is strongly reduced. This suggests that the phase of the junction is driven out of equilibrium by the gate. In order to confirm this out-of-equilibrium scenario, we investigate the switching dynamics. To address the gate-induced superconducting phase dynamics $[40,41]$, we measure the switching probability histograms for a different set of control parameters (e.g., $v, V_{G}$, and $T$ ). To do so, we repeatedly ( $M$ times) current bias the SNS junction with a sawtooth signal varying from 0 to $10 \mu \mathrm{A}$ and back at the frequency $v \in[17-277] \mathrm{Hz}$. The junction switches to a resistive state with a certain probability $P(I)$. We show in Fig. 3 the histograms obtained for different gate voltages, temperatures, and low-energy quasiparticle injection currents. The switching probability can be written as a function of the escape rate 
$\Gamma(I)$ as $[42,43]$

$$
P(I)=\frac{\Gamma(I)}{\dot{I}} \exp \left[-\int_{0}^{I} \frac{\Gamma\left(I^{\prime}\right)}{\dot{I}} d I^{\prime}\right]
$$

where $\dot{I}=d I / d t$ is the speed of the current bias ramp. In the thermally activated regime, the escape rate is given by $[40,41,44]$

$$
\Gamma_{\mathrm{T}}(I)=\frac{\omega_{A}(I)}{2 \pi} \exp \left(-\frac{\Delta U(I)}{k_{B} T_{\varphi}}\right)
$$

where $\Delta U(I)$ is the potential barrier defined by the tiltedwashboard potential, $\omega_{A}(I)$ is the current dependent plasma frequency, and $T_{\varphi}$ is the phase temperature, which can be higher than the time-averaged electron temperature $T_{e}$, due to out-of-equilibrium current noise (see Supplemental Material [35] and other references [45,46] for more details).

We first verify that the temperature dependence of the histograms is well captured by the thermal rate expression (3). The histograms [orange color in Fig. 3(a)] slightly broaden and shift to lower current values when the temperature is raised from $100 \mathrm{mK}$ up to roughly $325 \mathrm{mK}$. Above $330 \mathrm{mK}$ a sharpening of the histograms is observed as the phase dynamics changes from switching to diffusion because of dissipation $[47,48]$. The transition between these two regimes occurs at a temperature corresponding to the minigap, as expected, since the number of thermal quasiparticles in $\mathrm{N}$ is strongly increased for $k_{B} T>\Delta_{\min }$. In the restricted range of low temperatures, the histograms are fitted using the thermal rate given in Eq. (3). The critical current $I_{C}$ and the effective phase temperature $T_{\varphi}$ extracted from the fits are shown in Fig. 3(d). $I_{c}$ diminishes from 10 to $5 \mu \mathrm{A}$ while the phase temperature increases from 800 to $1200 \mathrm{mK}$. The same analysis can be done for quasiparticle injection from the tunnel junction where a thermal fit [Eq. (3)] reproduces the data using the parameters shown in Fig. 3(c). This comparison confirms that injecting low energy quasiparticles $(E<20 \mathrm{meV})$ is equivalent to raising the time-averaged temperature, $T_{e}$. Note that this tunnel current injection corresponds to a voltage $\left(V_{T} \in[2,20] \mathrm{mV}\right)$ across the tunnel junction higher than that used for the spectroscopic experiment $\left(V_{T}<0.65 \mathrm{mV}\right)$ presented above and for which the injected current was too small to perturb the switching mechanism.

Comparing the gate dependence to the temperature dependence [Fig. 3(a)], the same reduction of the mean switching current by gate voltage leads to much broader histograms $[17,25]$. We tentatively fit these histograms using Eq. (3). The quality of the fits is reasonable for most gate voltages but fails to explain histograms close to $V_{G}=4 \mathrm{~V}$ [see the boxed area at the top of Fig. 3(a)] where the supercurrent starts to diminish and the histograms are clearly nonthermal. Away from this transient regime, the fitting parameters are shown in Fig. 3(b). The fitted phase temperature rises to $10 \mathrm{~K}$ for the largest gate voltage $V_{G}=10 \mathrm{~V}$ studied here, which is at least ten times larger than the equilibrium phase temperature and much larger than $T_{e}$ as probed by tunneling spectroscopy and reported in Sec. III B. Note also that the increase in $I_{c}$ obtained from the fits is not physical but effectively compensates the huge increase in the width of histograms. Finally, we also used the tunnel probe to study the combined action of injecting low energy quasiparticles, equivalent to raising the effective temperature, and high energy electrons leaking from the gate (see Supplemental Material [35]). In conclusion, the fitting parameters using Eq. (3) are not physical and confirm that a more sophisticated model than overheating is necessary, especially for the transition regime $\left(V_{G} \in[3.2,5.2] \mathrm{V}\right)$ where the histograms cannot be fitted.

To do so, we assume, similarly to single photon detectors [34], that electrons coming from the gate generate temperature spikes of amplitude $T_{S}$ equivalent to hot spots. The effect of the high energy electrons leaking from the gate is modeled by a Poissonian distribution of the temperature spikes over time. This reflects the statistics of an independent flow of high energy electrons for small leakage currents. These spikes induce phase fluctuations and do not necessarily switch the junction to the dissipative state if damping is sufficiently high as corroborated by numerical solution of the resistively capacitively shunted junction (RCSJ) model (see Supplemental Material [35]). The escape rate associated with the gate leakage current is then given by $I_{L} / e$ multiplied by the escape probability that we choose here to be a thermal escape probability with a temperature $T_{S}$. With these assumptions, the total escape rate is

$$
\Gamma(I)=\Gamma_{\mathrm{T}}(I)+\frac{I_{L}}{e} \exp \left(-\frac{\Delta U(I)}{k_{B} T_{S}}\right) .
$$

For a more direct comparison of the data to Eq. (4) we extract the escape rates as a function of $I$ from the switching histograms inverting Eq. (2). We verified first that the escape rate is independent of the ramp frequency (which the histograms show; see the Supplemental Material [35] for details). The escape rates for different gate voltages are shown in Fig. 4 in the regime of weak leakage current, $V_{G} \in[3.2-5.2] \mathrm{V}$, corresponding to a negligible increase of the time-averaged electron temperature and very likely to a leakage current of uncorrelated electrons as supposed in Eq. (4). The log scale graph in Eq. 4(a) demonstrates that, first, the logarithm of the escape rates is nearly linear in current bias (thus exponential rates); second, they grow as the gate voltage/leakage current is raised; and, third, each curve seems shifted upwards. Using the second term of Eq. (4), we fit our data with the leakage current $I_{L}$, the spike temperature $T_{S}$, and $I_{C}$ as fitting parameters. For $V_{G}=0 \mathrm{~V}$ (purple dots) the data are well fitted (red line) using only the first term of Eq. (4) and the same parameters ( $I_{C}$ and $T_{\varphi}$ ) as for the histograms shown in Fig. 3. Increasing $V_{G}$ requires the second term of Eq. (4) which even becomes dominant. Using both terms in the fitting procedure perfectly reproduces our experimental data (gray dots and orange lines in Fig. 4). Importantly, whereas $I_{C}$ and $T_{S}$ allow us to tackle the full shape of the curve, $I_{L}$ fixes the shift between the curves. The values of $I_{L}$ obtained from the fits as a function of $V_{G}$ are reported as orange markers in Fig. 4(b). They follow well the leakage current measured with a room temperature current amplifier also shown in Fig. 4(b). The parameters $I_{C}$ and $T_{S}$ are shown in the inset of Fig. 4(a). Interestingly, we find a quasi-gate-independent $T_{S}$ which is very close to the inferred $T_{0}=1.5 \mathrm{~K}$ estimated at $V_{G}=5 \mathrm{~V}$ using the formula proposed at the end of Sec. III. 

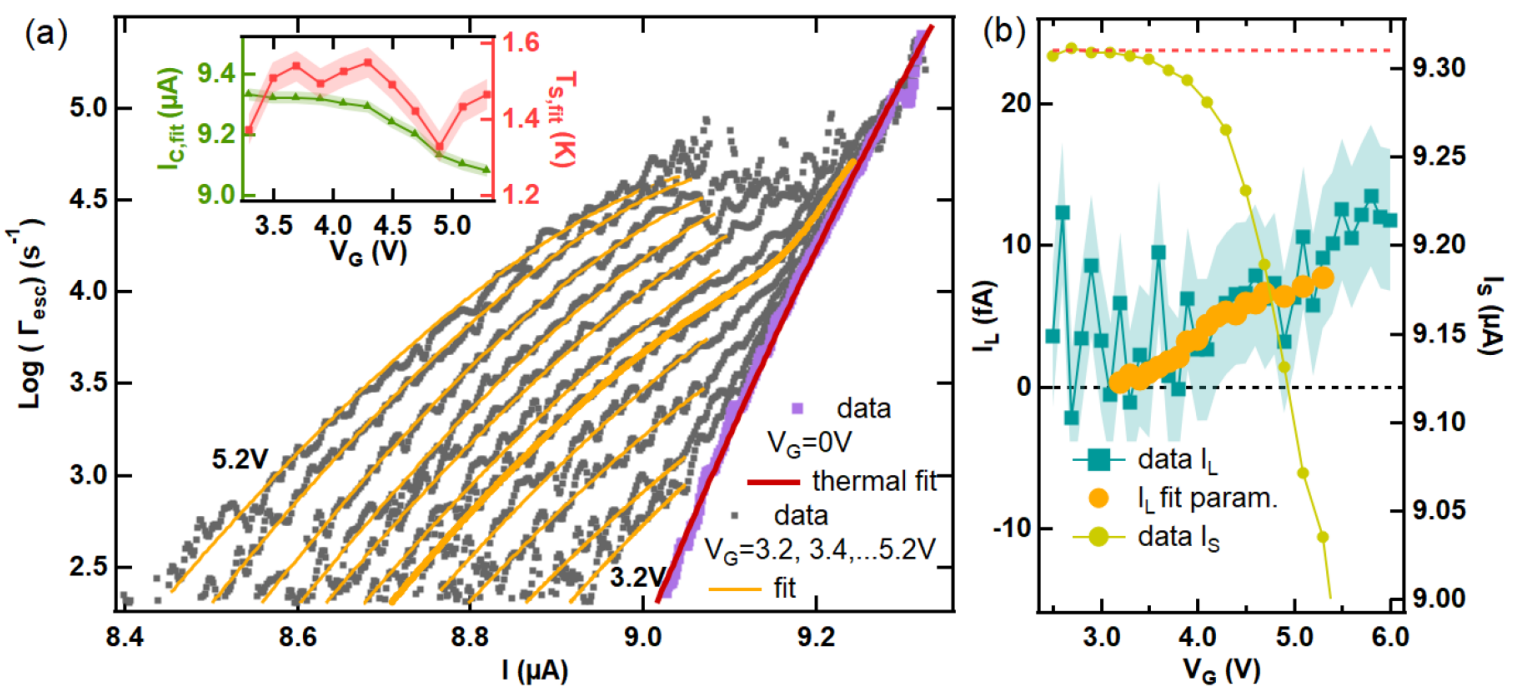

FIG. 4. Gate-assisted escape rates. (a) Escape rates for different $V_{G}$ as a function of current bias $I$ and the corresponding fits using Eqs. (2) and (4). Inset: Gate dependence of the fitting parameters $I_{C}$ and $T_{S}$. (b) Directly measured leakage current $I_{L}$, mean switching current $I_{S}$, and the fitting parameter $I_{L}$ extracted from the fits.

\section{DISCUSSIONS AND COMPARISON TO OTHER EXPERIMENTS}

Researchers from University of Lancaster [23] (see also [36]) recently measured the real and imaginary part of the microwave impedance of a gated Josephson weak link. They show that the field effect induces large $1 / f$ noise of the microwave impedance. This is consistent with gate-induced nonthermal phase fluctuations. Additionally, the spatially resolved suppression of the critical current reported in [24] has been attributed to current leakage from the gate. Even more recently it has been shown that the leakage current excites high energy phonons [25]. This observation is qualitatively consistent with the temperature spike scenario proposed here. It is however not clear yet which is the exact microscopic mechanism at the origin of the leakage current. Some recent studies report that it follows the Fowler-Nordheim model of electron field emission from a metal electrode $[11,23]$. In our case (see Supplemental Material [35]) but also in [25] this does not seem to be the case and it is more likely that the electrons flow either through the substrate or via surface states.

All these experiments point to out-of-equilibrium effects to explain the gate-induced reduction of the average critical current. We have here emphasized that high energy quasiparticles produce large phase fluctuations visible in the broadening of the switching histograms. Finally, if the leak rate is higher than the relaxation rate the temperature spikes overlap and a global overheating takes place [11]. From our experiment it is not possible to discriminate between charge and energy fluctuations induced by the leakage current, or in other words between the voltage and temperature pulses associated with absorption of hot electrons from the gate. However it is likely that energy imbalance is more relevant than charge imbalance simply because the estimated temperature spike in the normal wire resulting from a $5-\mathrm{eV}$ electron is $T_{0} \approx 1.5 \mathrm{~K}$, which sets an energy scale much larger than the chemical potential change that we estimate to $\delta \mu \approx e /\left(n_{\mathrm{Cu}} V_{\mathrm{Cu}}\right) \approx 10^{-8} \mathrm{eV}$ where $n_{\mathrm{Cu}}$ is the electron density of copper and $V_{\mathrm{Cu}}$ is its volume.

\section{CONCLUSION}

To conclude, we have performed an experiment to probe the field effect in a metallic Josephson junction by two complementary means, tunneling spectroscopy and switching histograms. Our experiment reveals that the gate-controllable switching current is triggered by high energy electrons leaking from the gate to the Josephson junction. Their effect is not equivalent to overheating but instead to locally induced large energy fluctuations which translate as large phase fluctuations visible in the switching histograms. From the escape rates obtained from the histograms, we could extract the leakage current in good agreement with direct measurements. These findings clarify the local dynamics of high energy quasiparticles and shed light on the complex mechanisms at play in gated metallic Josephson junctions.

\section{ACKNOWLEDGMENTS}

We thank F. Giazotto, B. Reulet, and C. Di Giorgio for fruitful discussions and F. Paolucci and F. Giazotto for providing some SNS samples at the beginning of this work. This research has been funded by a ANR JCJC (SPINOES) grant from the Agence Nationale de la Recherche.
[1] R. M. Lutchyn, L. I. Glazman, and A. I. Larkin, Kinetics of the superconducting charge qubit in the presence of a quasi-particle, Phys. Rev. B 74, 064515 (2006). 
[2] M. Zgirski, L. Bretheau, Q. Le Masne, H. Pothier, D. Esteve, and C. Urbina, Evidence for Long-Lived Quasiparticles Trapped in Superconducting Point Contacts, Phys. Rev. Lett. 106, 257003 (2011).

[3] D. S. Golubev and A. D. Zaikin, Thermally activated phase slips in superconducting nanowires, Phys. Rev. B 78, 144502 (2008).

[4] J. M. Martinis, M. Ansmann, and J. Aumentado, Energy Decay in Superconducting Josephson-Junction Qubits from Nonequilibrium Quasiparticle Excitations, Phys. Rev. Lett. 103, 097002 (2009).

[5] A. P. Vepsäläinen, A. H. Karamlou, J. L. Orrell, A. S. Dogra, B. Loer, F. Vasconcelos, D. K. Kim, A. J. Melville, B. M. Niedzielski, J. L. Yoder, S. Gustavsson, J. A. Formaggio, B. A. VanDevender, and W. D. Oliver, Impact of ionizing radiation on superconducting qubit coherence, Nature (London) 584, 551 (2020).

[6] L. Cardani, F. Valenti, N. Casali, G. Catelani, T. Charpentier, M. Clemenza, I. Colantoni, A. Cruciani, G. D’Imperio, L. Gironi, L. Grünhaupt, D. Gusenkova, F. Henriques, M. Lagoin, M. Martinez, G. Pettinari, C. Rusconi, O. Sander, C. Tomei, A. V. Ustinov et al. Reducing the impact of radioactivity on quantum circuits in a deep-underground facility, Nat. Commun. 12, 2733 (2021).

[7] D. Beckmann, H. B. Weber, and H. V. Löhneysen, Evidence for Crossed Andreev Reflection in Superconductor-Ferromagnet Hybrid Structures, Phys. Rev. Lett. 93, 197003 (2004).

[8] P. Cadden-Zimansky, Z. Jiang, and V. Chandrasekhar, Charge imbalance, crossed Andreev reflection and elastic co-tunnelling in ferromagnet/superconductor/normal-metal structures, New J. Phys. 9, 116 (2007).

[9] A. Kleine, A. Baumgartner, J. Trbovic, D. S. Golubev, A. D. Zaikin, and C. Schönenberger, Magnetic field and contact resistance dependence of non-local charge imbalance, Nanotechnology 21, 274002 (2010).

[10] C. H. L. Quay, D. Chevallier, C. Bena, and M. Aprili, Spin imbalance and spin-charge separation in a mesoscopic superconductor, Nat. Phys. 9, 84 (2013).

[11] L. D. Alegria, C. G. L. Bøttcher, A. K. Saydjari, A. T. Pierce, S. Hwan Lee, S. P. Harvey, U. Vool, and A. Yacoby, High-energy quasiparticle injection into mesoscopic superconductors, Nat. Nanotechnol. 16, 404 (2021).

[12] G. De Simoni, F. Paolucci, P. Solinas, E. Strambini, and F. Giazotto, Metallic supercurrent field-effect transistor, Nature Nanotechnol. 13, 802 (2018).

[13] F. Paolucci, G. De Simoni, E. Strambini, P. Solinas, and F. Giazotto, Ultra-efficient superconducting dayem bridge fieldeffect transistor, Nano Lett. 18, 4195 (2018).

[14] G. De Simoni, F. Paolucci, C. Puglia, and F. Giazotto, Josephson field-effect transistors based on all-metallic $\mathrm{Al} / \mathrm{Cu} / \mathrm{Al}$ proximity nanojunctions, ACS Nano 13, 7871 (2019).

[15] F. Paolucci, G. De Simoni, P. Solinas, E. Strambini, N. Ligato, P. Virtanen, A. Braggio, and F. Giazotto, Magnetotransport Experiments on Fully Metallic Superconducting Dayem-Bridge Field-Effect Transistors, Phys. Rev. Applied 11, 024061 (2019).

[16] F. Paolucci, F. Vischi, G. De Simoni, C. Guarcello, P. Solinas, and F. Giazotto, Field-effect controllable metallic josephson interferometer, Nano Lett. 19, 6263 (2019).

[17] C. Puglia, G. De Simoni, and F. Giazotto, Electrostatic Control of Phase Slips in Ti Josephson Nanotransistors, Phys. Rev. Applied 13, 054026 (2020).
[18] M. Rocci, G. De Simoni, C. Puglia, D. Degli Esposti, E. Strambini, V. Zannier, L. Sorba, and F. Giazotto, Gatecontrolled suspended titanium nanobridge supercurrent transistor, ACS Nano 14, 12621 (2020).

[19] F. Paolucci, G. De Simoni, P. Solinas, E. Strambini, C. Puglia, N. Ligato, and F. Giazotto, Field-effect control of metallic superconducting systems, AVS Quantum Sci. 1, 016501 (2019).

[20] A. F. Morpurgo, T. M. Klapwijk, and B. J. van Wees, Hot electron tunable supercurrent Appl. Phys. Lett. 72, 966 (1998).

[21] S. B. Lee, G. D. Hutchinson, D. A. Williams, D. G. Hasko, and H. Ahmed, Superconducting nanotransistor based digital logic gates, Nanotechnology 14, 188 (2003).

[22] A. Wagner, L. Ranzani, G. Ribeill, and T. A. Ohki, Demonstration of a superconducting nanowire microwave switch, Appl. Phys. Lett. 115, 172602 (2019).

[23] I. Golokolenov, A. Guthrie, S. Kafanov, Yu. A. Pashkin, and V. Tsepelin, On the origin of the controversial electrostatic field effect in superconductors, Nat. Commun. 12, 2747 (2021).

[24] M. F. Ritter, A. Fuhrer, D. Z. Haxell, S. Hart, P. Gumann, H. Riel, and F. Nichele, A superconducting switch actuated by injection of high-energy electrons, Nat. Commun 12, 1266 (2021).

[25] M. F. Ritter, N. Crescini, D. Z. Haxell, M. Hinderling, H. Riel, C. Bruder, A. Fuhrer, and F. Nichele, On the role of out-of-equilibrium phonons in gated superconducting switches, arXiv:2106.01816 (2021).

[26] H. le Sueur, P. Joyez, H. Pothier, C. Urbina, and D. Estève, Phase Controlled Superconducting Proximity Effect Probed by Tunneling Spectroscopy, Phys. Rev. Lett. 100, 197002 (2008).

[27] I. Giaever, Energy Gap in Superconductors Measured by Electron Tunneling, Phys. Rev. Lett. 5, 147 (1960).

[28] H. Pothier, S. Guéron, Norman O. Birge, D. Esteve, and M. H. Devoret, Energy Distribution Function of Quasiparticles in Mesoscopic Wires, Phys. Rev. Lett. 79, 3490 (1997).

[29] F. Marsili, M. J. Stevens, A. Kozorezov, V. B. Verma, Colin Lambert, J. A. Stern, R. D. Horansky, S. Dyer, S. Duff, D. P. Pappas, A. E. Lita, M. D. Shaw, R. P. Mirin, and S. W. Nam, Hotspot relaxation dynamics in a current-carrying superconductor, Phys. Rev. B 93, 094518 (2016).

[30] A. M. Kadin and M. W. Johnson, Nonequilibrium photoninduced hotspot: A new mechanism for photodetection in ultrathin metallic films, Appl. Phys. Lett. 69, 3938 (1996).

[31] G. N. Goltsman, O. Okunev, G. Chulkova, A. Lipatov, A. Semenov, K. Smirnov, B. Voronov, A. Dzardanov, C. Williams, and R. Sobolewski, Picosecond superconducting single-photon optical detector, Appl. Phys. Lett. 79, 705 (2001).

[32] A. Engel and A. Schilling, Numerical analysis of detectionmechanism models of superconducting nanowire single-photon detector, J. Appl. Phys. 114, 214501 (2013).

[33] A. N. McCaughan, V. B. Verma, S. M. Buckley, J. P. Allmaras, A. G. Kozorezov, A. N. Tait, S. W. Nam, and J. M. Shainline, A superconducting thermal switch with ultrahigh impedance for interfacing superconductors to semiconductors, Nat. Electronics 2, 451 (2019).

[34] E. D. Walsh, W. Jung, G.-H. Lee, D. K. Efetov, B.-I. Wu, K.F. Huang, T. A. Ohki, T. Taniguchi, K. Watanabe, P. Kim, D. Englund, and K. C. Fong, Josephson junction infrared singlephoton detector, Science 372, 409 (2021).

[35] See Supplemental Material at http://link.aps.org/supplemental/ 10.1103/PhysRevResearch.3.043169 for additional data and 
discussions regarding the experimental setup, numerical simulations, switching histograms, and the combined action of low and high energy electron injection.

[36] G. Catto, W. Liu, S. Kundu, V. Lahtinen, and M. Möttönen, Microwave response of a metallic superconductor subject to a high-voltage gate electrode, arXiv:2105.08322 (2021).

[37] C. Puglia, G. De Simoni, and F. Giazotto, Gate control of superconductivity in mesoscopic all-metallic devices, Materials 14, 1243 (2021).

[38] A. G. Kozorezov, A. F. Volkov, J. K. Wigmore, A. Peacock, A. Poelaert, and R. den Hartog, Quasiparticle-phonon downconversion in nonequilibrium superconductors, Phys. Rev. B 61, 11807 (2000).

[39] M. Lenander, H. Wang, R. C. Bialczak, E. Lucero, M. Mariantoni, M. Neeley, A. D. O'Connell, D. Sank, M. Weides, J. Wenner, T. Yamamoto, Y. Yin, J. Zhao, A. N. Cleland, and J. M. Martinis, Measurement of energy decay in superconducting qubits from nonequilibrium quasiparticles, Phys. Rev. B 84, 024501 (2011).

[40] W. C. Stewart, Current-voltage characteristics of Josephson junctions, Appl. Phys. Lett. 12, 277 (1968).

[41] D. E. McCumber, Effect of ac impedance on dc voltagecurrent characteristics of superconductor weak-link junctions, J. Appl. Phys. 39, 3113 (1968).
[42] J. Kurkijärvi, Intrinsic fluctuations in a superconducting ring closed with a Josephson junction, Phys. Rev. B 6, 832 (1972).

[43] T. A. Fulton and L. N. Dunkleberger, Lifetime of the zerovoltage state in Josephson tunnel junctions, Phys. Rev. B 9, 4760 (1974).

[44] H. A. Kramers, Brownian motion in a field of force and the diffusion model of chemical reactions, Physica 7, 284 (1940).

[45] T. T. Heikkilä, J. Särkkä, and F. K. Wilhelm, Supercurrentcarrying density of states in diffusive mesoscopic Josephson weak links, Phys. Rev. B 66, 184513 (2002).

[46] J. Basset, M. Kuzmanović, P. Virtanen, T. T. Heikkilä, J. Estève, J. Gabelli, C. Strunk, and M. Aprili, Nonadiabatic dynamics in strongly driven diffusive Josephson junctions, Phys. Rev. Research 1, 032009(R) (2019).

[47] V. M. Krasnov, T. Bauch, S. Intiso, E. Hürfeld, T. Akazaki, H. Takayanagi, and P. Delsing, Collapse of Thermal Activation in Moderately Damped Josephson Junctions, Phys. Rev. Lett. 95, 157002 (2005).

[48] J. Mannik, S. Li, W. Qiu, W. Chen, V. Patel, S. Han, and J. E. Lukens, Crossover from Kramers to phase-diffusion switching in moderately damped Josephson junctions, Phys. Rev. B 71, 220509(R) (2005). 